\title{
1 Genetic analysis of 17 Y-STR loci in Han population from Shandong Province in
}

2

3 Jing $\mathrm{Xu}^{1 \dagger}$, Liming $\mathrm{Li}^{2,3 \dagger}$, Lanhai $\mathrm{Wei}^{2,3}$, Zhiyi $\mathrm{Nie}^{4}$, Shuping Yang ${ }^{2,3}$, Mingying $\mathrm{Xia}^{2,3}$,

4 Teng Ma ${ }^{2,3}$, Hui Sun ${ }^{2,5}$, Xueying Zhao ${ }^{3}$, Yuan Ping ${ }^{3}$, Huaigu Zhou ${ }^{3}$, Fuzhong Xue ${ }^{6}$, $5 \quad$ Ziqin Zhao ${ }^{1}$, Li Jin ${ }^{2,3}$, Shilin $\mathrm{Li}^{2,3 *}$

6

$7 \quad{ }^{1}$ Department of Forensic Medicine, Shanghai Medicine College, Fudan University, 8 Shanghai, China

$9 \quad{ }^{2}$ Ministry of Education Key Laboratory of Contemporary Anthropology and State Key

\section{East China}

Laboratory of Genetic Engineering, Collaborative Innovation Center for Genetics and

Development, School of Life Sciences, Fudan University, Shanghai, China

${ }^{3}$ Shanghai Public Security Bureau-Fudan University Joint Laboratory of Human

Biology and Forensic Techniques for Crime Scenes, Shanghai Research Institute of Criminal Science and Technology, Shanghai Key Laboratory of Crime Scene Evidence, Key Laboratory of Forensic Evidence and Science Technology, Ministry of Public Security, Shanghai, China

${ }^{4}$ Forensic Science Center, East China University of Political Science and Law, Shanghai, China

${ }^{5}$ Institute of Forensic Science, Ministry of Public Security, Key Laboratory of Forensic Genetics, Ministry of Public Security, Beijing, China

${ }^{6}$ School of Public Health, Shandong University, Jinan, Shandong, China.

\section{${ }^{\dagger}$ The two authors contributed equally to the work.}


24 "Corresponding author:

25 Dr. Shilin Li, School of Life Sciences, Fudan University,

262005 Songhu Road, Shanghai 200438, China

27 Tel: 8602151630602

28 Fax: 8602151630607

29 Email: lishilin@,fudan.edu.cn

30 


\section{Genetic analysis of 17 Y-STR loci in Han population from}

\section{Shandong Province in EastChina}

Dear Editor, Shandong Provinceis located in East China, including two sub-provincial and fifteen prefecture-level cities (Fig. S1). According to the 2009 census, the population of Shandongreached 95,793,065, andHan made up the largest ethnic group.

In the present study, a total of734unrelated male Han Chinese individuals were collected from ShandongProvince. These samples were collected from twelve sub-provincial or prefecture-level cities, including Jinan $(n=59)$, Binzhou $(n=30)$, Dezhou $(n=114)$, Dongying $(n=76)$, Heze $(n=29)$, Jining $(n=41)$, Linyi $(n=122)$, Rizhao (n=29), Tai'an ( $\mathrm{n}=21)$, Weifang $(\mathrm{n}=21)$, Yantai $(\mathrm{n}=122)$ and Zibo $(\mathrm{n}=70)$.Peripheral blood samples were collected from these individuals after acquiring their informed consent. Genomic DNA was extracted using the phenol-chloroform method.A total of 17 Y-STRs were analyzed, including DYS19, DYS389I, DYS389II, DYS390, DYS391, DYS392, DYS393, DYS437, DYS438, DYS439, DYS448, DYS456, DYS458, DYS635, YGATAH4 and DYS385a/b. Polymerase chain reaction (PCR) was performed using the AmpFlSTR Yfiler ${ }^{\mathrm{TM}}$ PCR Amplification kit (Thermo Fisher Scientific Company, Carlsbad, USA) in the GeneAmp PCR System 9700 (Thermo Fisher Scientific Company) according to the manufacturer's instruction. PCR products were separated by capillary electrophoresis in ABI PRISM 3130xL Genetic Analyzer (Thermo Fisher Scientific Company). The GeneMapper ID software 
v3.2 (Thermo Fisher Scientific Company) was usedfor genotype assignment. DNA typing and assignment of nomenclature were based on the ISFG recommendations $[1,2]$.

We estimated pairwise Rst between twelve Han populations of Shandongusing Arlequin v3.5 (10,000 permutations)[3]. In this analysis, DYS385 a/b was excluded, and DYS389B was obtained by subtracting the allele of DYS389I from that of DYS389II.The haplotype frequencywas estimated using direct counting method. Haplotype diversitywasestimated as described byNei[4]: HD $=(n / n-1)\left(1-\sum p_{i}^{2}\right)$, where $\mathrm{n}$ is the sample size and $\mathrm{p}_{\mathrm{i}}$ is the frequency of the ith haplotype.Moreover, pairwise Rst was estimated between Shandong Han and other Chinese populations in previous reportsusing Arlequin v3.5[3], and the results were visualized by multidimensional scaling plot (MDS) using R v3.1.2 (http://www.r-project.org).

$\underline{\text { We observed significant difference between Dezhou and Linyi after Bonferroni }}$ correction $(p=0.05 / 66=0.00075758,66$ arethe number of the test). Significant differences were also observed between Zibo and the other six Shandong Han populations includingBinzhou, Dezhou, Jinan, Linyi, Rizhao and Yantai(Table S1). The results showed that the haplotype frequency of Zibo was slightly different from other Han populations in Shandong of the present study, and thismay require further evaluation on larger dataset.

A total of 701 different haplotypes were observed among all individuals of Shandong Han population, of which 676 were unique, 21 were shared by two individuals, 3 were shared bythree individuals and 1 were shared byseven individuals 
(Table S2). The haplotype diversityreached0.99981.

We estimated pairwise Rst between the Han population of Shandong in this study and other reports involving Chinese populations. A total of fourteen Chinese Han [5-13] and twelve ethnic populations [6, 14-21] were obtained from earlier reports. Among them, two Han populations were sampled from Shandong Province [7, 13]. Overall, 8,056 reference samples were analyzed. Figure S2 showed that Shandong Han is genetically close to other Han populations and to Manchu, Mongolian, and Xibe.As shown in Table S3,ShandongHan of the present study had no significant difference between Shandong,China [Han], whereasshowed significant difference between Jining, China $[\mathrm{Han}]$ after Bonferroni correction $(p=0.05 / 351=$ $\underline{0.00014245,351 \text { arethe number of the test). In addition, we observed that Shandong }}$ Han of the present study showed significant differences between the other five Chinese Han populations (Jiangsu, China [Han], Zhejiang, China [Han], Luzhou, China [Han], Shanxi, China [Han] and Henan, China [Han]). Significant differences were also observed between Shandong Han and nearly all Chinese ethnic populations except Manchu and Xibe.

In conclusion, we report 17 Y-STR loci's haplotype frequencies and forensic parameters of the Han population in Shandong Province. We believe the data is valuable for both forensics and population genetics.

Our data has been submitted to YHRD, and received the accession number: Shandong, China [Han], n=734, YA004081.

The study was supported by grants from the National Science Foundation of 
China (31271338), Project of Chinese Ministry of Education (113022A) and National

High Technology Research and Development Program (2012AA021802).

Thestudy follows the new guidelines for publication of population data requested by the journal[22, 23].

\section{Reference}

[1] Bar W, Brinkmann B, Budowle B, Carracedo A, Gill P, Lincoln P, Mayr W, Olaisen B: DNA recommendations - Further report of the DNA Commission of the ISFH regarding the use of short tandem repeat systems. Int J Legal Med 1997, 110(4):175-176.

[2] Olaisen B, Bar W, Brinkmann B, Budowle B, Carracedo A, Gill P, Lincoln P, Mayr WR, Rand S: DNA recommendations 1997 of the International Society for Forensic Genetics. Vox Sang 1998, 74(1):61-63.

[3] Excoffier L, Lischer HEL: Arlequin suite ver 3.5: a new series of programs to perform population genetics analyses under Linux and Windows. Mol Ecol Resour 2010, 10(3):564-567.

[4] Nei M: Molecular evolutionary genetics. New York: Columbia University Press; 1987, pp. 176-179.

[5] Li Liming, Ma Teng, Xu Jing, Yang Yajun, Yang Shuping, Xia Mingying, Sun Hui, Zhao Xueying, Ping Yuan, Zhou Huaigu, Xie Jianhui, Zhao Ziqin, Jin Li, Li Shilin: Genetic analysis of 17 Y-STR loci in Han population from Gansu Province in northwestern China. Forensic Sci Int-Genet 2015, 19:134-135.

[6] Shu Lili, Li Liming, Yu Ge, Yu Binxia, Liu Yuchi,Li Shilin, Jin Li, Yan Shi: Genetic analysis of 17 Y-STR loci in Han, Dong, Miao and Tujia populations from Hunan province, central-southern China. Forensic Sci Int-Genet 2015, 07. 007

[7] Li Liming, Yu Ge, Li Shilin, Jin Li, Yan Shi: Genetic analysis of 17 Y-STR loci from 1,019 individuals of six Han populations in East China. Forensic Sci Int-Genet 2015, 20:101-102.

[8] Yang YY, Yuan WL, Guo F, Jiang XH: Population data of 17 Y-STR loci in Nanyang Han population from Henan Province, Central China. Forensic Sci Int-Genet 2014, 13:145-146.

[9] Bing L, Liang WB, Pi JH, Zhang DM, Yong D, Luo HB, Zhang LS, Lin Z: Population genetics for 17 Y-STR loci(AmpFISTR (R) Y-filerTM) in Luzhou Han ethnic group. Forensic Sci Int-Genet 2013, 7(2):E23-E26.

[10] Liu Y, Liao LC, Gu MB, Ye Y: Population genetics for 17 Y-STR loci in a Chinese Han population sample from Mudanjiang city, Northeast China. Forensic Sci Int-Genet 2014, 13:E16-E17.

[11] Bai RF, Zhang Z, Liang QZ, Lu D, Yuan L, Yang X, Shi MS: Haplotype diversity of 17 Y-STR loci in a Chinese Han population sample from Shanxi Province, Northern China. Forensic Sci Int-Genet 2013, 7(1):214-216.

[12] Shi Meisen, Liu Yaju, Zhang Juntao, Bai Rufeng, Lv Xiaojiao, Ma Shuhua: Analysis of 24 Y 
chromosomal STR haplotypes in a Chinese Hanpopulation sample from Henan Province, Central China. Forensic Sci Int-Genet 2015, 17:83-86.

[13] Wang Dan, Liu Faqin, Kong Lingxue, Yuan Zhenyu, Chen Guang, Ye Jian: Population data of 17 Y-STR haplotypes in Jining Hanpopulation from Shandong province, East China. Forensic Sci Int-Genet 2015.05.017.

[14] Luo Haibo, Song Feng, Zhang Lushun, Hou Yiping: Genetic polymorphism of 23 Y-STR loci in the Zhuang minoritypopulation in Guangxi of China. Int J Legal Med2015, 129(4):737-738.

[15] Guo F: Population genetics for 17 Y-STR loci in Mongolian ethnic minority from Liaoning Province, Northeast China. Forensic Sci Int-Genet 2015, 17:153-154.

[16] Guo F, Zhang L, Jiang XH: Population genetics of 17 Y-STR loci in Xibe ethnic minority from Liaoning Province, Northeast China. Forensic Sci Int-Genet 2015, 16:86-87.

[17] Li KL, Kai L, Yuming M: Y chromosome STR haplotypes of Tibetan living Tibet lassa. Forensic Scilnt 2007, 172(1):79-83.

[18] Zhu BF, Wu YM, Shen CM, Yang TH, Deng YJ, Xun X, Tian YF, Yan JC, Li T: Genetic analysis of 17 Y-chromosomal STRs haplotypes of Chinese Tibetan ethnic group residing in Qinghai province of China. Forensic Scilnt 2008, 175(2-3):238-243.

[19] Shan WJ, Ablimit A, Zhou WJ, Zhang FC, Ma ZH, Zheng XF: Genetic polymorphism of 17 Y chromosomal STRs in Kazakh and Uighur populations from Xinjiang, China. Int J Legal Med 2014, 128(5):743-744.

[20] He J, Guo F: Population genetics of 17 Y-STR loci in Chinese Manchu population from Liaoning Province, Northeast China. Forensic Sci Int-Genet 2013, 7(3):E84-E85.

[21] Wu FC, Ho CW, Pu CE, Hu KY, Willuweit S, Roewer L, Liu DH: Y-chromosomal STRs haplotypes in the Taiwanese Paiwan population. Int J Legal Med 2011, 125(1):39-43.

[22] Carracedo A, Butler JM, Gusmao L, Linacre A, Parson W, Roewer L, Schneider PM: New guidelines for the publication of genetic population data. Forensic Sci Int-Genet 2013, 7(2):217-220.

[23] Carracedo A, Butler JM, Gusmao L, Linacre A, Parson W, Roewer L, Schneider PM: Update of the guidelines for the publication of genetic population data. Forensic Sci Int-Genet 2014, 10:A1-A2. 